\title{
Research on Energy Industry Strategy Based on Intelligent Digital Upgrading
}

\author{
Zhang zhongpeng ${ }^{1, *}$, Feng Lei $^{2}$, Zheng zhengbing ${ }^{1}$, and Wang guibao ${ }^{1}$ \\ ${ }^{1}$ School of Physics and Telecommunication Engineering, Shaanxi University of Technology, Hanzhong, China \\ ${ }^{2}$ Personnel Department, Shaanxi University of Technology, Hanzhong, China
}

\begin{abstract}
The global energy supply and demand are constantly changing, and toward the leapfrog development of digital energy. How to further accelerate the pace of energy digital transformation is the direction that many scholars have been exploring. Starting from the background of international energy strategy, this article analyzes and researches new products of energy strategy digitization, energy data center intelligence and digital energy strategy, and then discusses the development trend and significance of intelligence in the future energy field. It aims to use digital technology and intelligent concepts to promote the in-depth integration of energy and modern information technology, promote the transformation of energy production management and marketing models, and reshape the energy industry chain, supply chain and value chain to promote the drawing of an intelligent blueprint in the energy field.
\end{abstract}

\section{Introduction}

At present, the digital economy is becoming the growth engine of the global economy. The Oxford Institute for Economic Research report shows that the long-term return of digital technology is 6.7 times that of nondigital technology. The "White Paper on China's Digital Economy Development (2020)" of the China Academy of Information and Communications Technology also shows that in 2019, the value added of China's digital economy reached 35.9 trillion yuan, accounting for $36.2 \%$ of GDP. However, with the vigorous development of the digital economy, new and old problems such as high energy consumption of digital infrastructure, long construction period, and low efficiency of operation and maintenance have gradually surfaced. Therefore, energy conservation and emission reduction actions and digital transformation are becoming more and more intense. A new round of technological revolution and industrial transformation in the energy sector is about to emerge. A picture scroll of a big era is slowly unfolding to us. Faced with such a brand-new subject, only by finding the right direction can efforts be made more valuable[1].

In this context, many studies start from the overall situation of the industry to the system architecture, and then to power generation, storage, electricity and even all aspects of security and credibility, focusing on accelerating energy digitization and solving energy problems in economic development to provide guidance and methodology, and to lead the new pattern of green intelligence in the world. In a sense, this also reflects a new human understanding of digital energy - standing in a broader perspective of the green and sustainable development of human society, the energy industry and the digital industry 's mutual empowerment, rather than just thinking and exploring from their own business growth to make greater contributions and create greater value.

\section{Research on the development of energy strategy data}

From 2007 to 2020, relevant domestic and foreign studies have fully demonstrated that the integrated energy system (IES) has become the choice for future energy low-carbon and high-efficiency applications. Based on the important characteristics of time complexity, space complexity, and behavioral complexity of the energy system, it faces problems such as difficulty in data integrity, difficulty in obtaining samples, and difficulty in status analysis during safe and efficient operation. In view of the above three difficulties, we will carry out targeted research in three aspects, namely, understanding data characteristics, mining sample associations, and studying and judging status differences. In terms of understanding the characteristics of data, a more detailed study can be carried out on the data matrix model and the network result model. In the sample acquisition process, attention should be paid to the generation of small samples, and the generator training process and generator network structure can be optimized. Finally, try to complete the state analysis without prior knowledge of the system. In order to solve the problem of data completeness, it is necessary to carry out unified data measurement, optimize data sorting, and grasp the trend of data; to solve the problem of difficult sample acquisition, it is necessary to implement sample

* Corresponding author: anthony@snut.edu.cn 
physical constraints, optimize the network training process and network structure parameters; to solve the state Analyzing difficult problems requires special comprehensive analysis, mastering data statistical indicators, and conducting zero-sample self-learning.

With the advent of the "operating system as a service" era, it is an extremely important opportunity for the energy industry in the process of intelligent transformation and upgrading. Energy companies are the first to take the lead, achieve leapfrog development and enhance comprehensive competitiveness. Great opportunity [2]. The new combination of software and hardware will optimize the efficiency of the energy industry and promote the informatization transformation of the energy industry. The energy industry has a significant impact on the macro economy, and the transformation of the energy industry will effectively promote the improvement of the overall economic quality. For large energy companies, the consolidation of IT infrastructure will help build a reliable energy supply, production, and transmission system. Effective IT investment in the Internet of Things, big data analysis, etc. will bring considerable efficiency improvements. Some energy companies have improved the upgrade and deployment of the company's new-generation IT architecture, further improved the operating efficiency of the company's business system, and better supported the stable operation of the company's business system in the complex and changeable Internet environment. Efficiently build an information application platform, create a new high-performance desktop system environment, and help enterprises to deploy Internet transformation. For example, the construction of smart grids is an important aspect of energy transformation. It improves the targeted supply capacity of the energy network, meets the diverse needs of users, and helps build an efficient and smart energy system and realize the transformation and upgrading of the industry.

\section{Research on the development of energy strategy data}

In the process of promoting data center intelligence and creating a new green model of energy saving and consumption reduction, Data Center Pass innovatively reconstructs the power supply system, temperature control system, operation and maintenance and architecture to achieve intelligent construction, centralized management, unified operation and maintenance and high reliability of the server room. To further solve the problems of the majority of ecological partners in the process of building data center intelligence, many ecological partners and industry representatives are jointly discussing how to take a multi-pronged approach from the construction, management, operation and maintenance levels to effectively improve resource utilization and accelerate the realization of green data centers.

As emerging technologies such as cloud computing, Internet of Things, and artificial intelligence have sprung up and accelerated their popularity, the future human society will evolve into a society of perception, interconnection of all things, and intelligence of all things. The ICT infrastructure that carries these key technologies will Become the cornerstone of the smart world, and the data center is a key link in the entire ICT ecological chain. To this end, a large number of scholars are advancing along the path of comprehensive cloudification of data centers, focusing on equipment, networks, services, operations, etc., to make the data center infrastructure more reliable, more efficient, and simpler, thereby effectively supporting the entire industry transformation Upgrade [3].

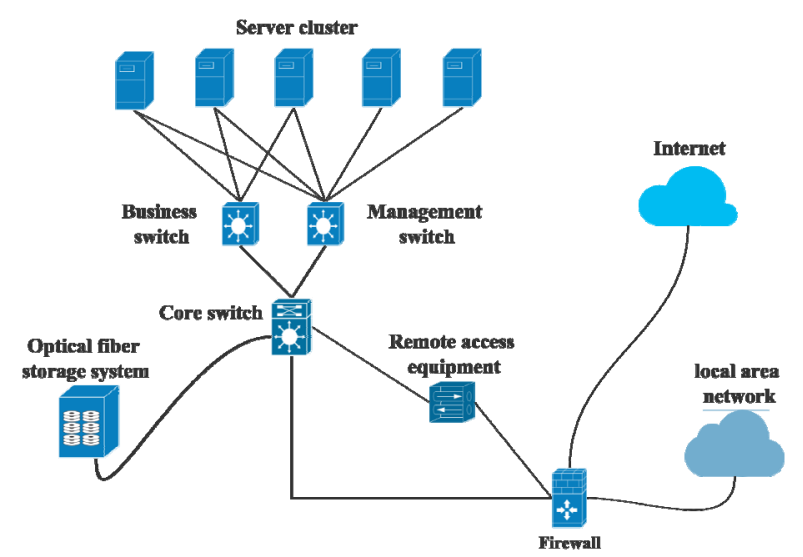

Figure 1. Topology diagram of energy data center

\section{Analysis of New Products of Digital Energy Strategy}

With the continuous advancement of the construction of intelligent energy, the achievements of smart energy represented by virtual power plants have gradually emerged. In the future, more silent distributed resources will be awakened. Virtual power plants are the realization of multi-form resources in the smart energy ecology. The key part of "gathering sand into a tower, wisely responding". On this basis, we must continue to consolidate our background advantages, rely on conceptual innovation and technological breakthroughs, optimize and improve the virtual power plant system, connect upstream and downstream enterprises in the entire industry chain, and create an active, convenient, and shared virtual power plant ecosystem, which is intelligent for the energy industry. Chemical transformation continues to inject new kinetic energy.

\subsection{Create "Poetry and Distance" of Energy Transition}

Since the beginning of the 21 st century, with the rapid economic development, energy structure optimization, green development and other issues have become increasingly urgent. The energy transition has presented unprecedented challenges to the function and operation of the power grid. Distributed energy construction has become the main direction of energy development. Virtual power plant, as a regional energy aggregation mode that can realize a large number of distributed 
energy access to the grid, has become the "poem and distant place" of energy transition.

The virtual power plant does not have the form of a physical power plant, but has the function of a physical power plant. It organically combines distributed generating sets, controllable loads, and energy storage facilities. It regulates and manages distributed energy through supporting regulation and communication technologies, and participates in power market transactions and grid operations in the form of special power plants. Based on a deep understanding of energy development and power grid business, relying on practical experience in information communication, smart energy, electric vehicles and other fields, fully mobilizing superior resources, actively exploring and practicing virtual power plant construction, has become the core of research in the field of virtual power plants in the power industry.

\subsection{Key technologies to improve performance}

"Aggregation" and "communication" are the key technologies of virtual power plants. Different types of distributed resources such as distributed power sources, distributed energy storage, controllable loads, and charging piles are like scattered "sand". The virtual power plant technology gathers and utilizes these "sands", integrates and collaborates, and forms a huge

"tower" . Communication technology is an important channel for virtual power plants to conduct resource management, data acquisition and monitoring, and information interaction. The innovative use of $5 \mathrm{G}$ communication technology, using its advantages of low latency, high reliability, and wide coverage, can solve the network bottleneck problem of virtual power plants and meet the large parallel and real-time control of distributed energy, energy storage sites and flexible loads. To realize the real-time collection and control of distributed resources, and improve the safety, accuracy and timeliness of virtual power plant operation [4].

Aggregation and coordination optimization is an important support for virtual power plants to regulate resources and improve grid operation efficiency. Therefore, relying on intelligent control technology, adopting refined modeling and efficient solving algorithms, facing the power control center, providing a multi-cycle, multi-objective, multi-dimensional, multiscenario comprehensive grid operation control solution has become the mainstream of the industry. At the same time, the core intelligent analysis and forecasting technology, through in-depth exploration of the related influences and changing laws of various factors, provides fine-grained and accurate power and load forecasting, and provides decision support for virtual power plants to participate in power dispatching operations.

\subsection{Build a platform-based operation model}

The management and control platform is the "most powerful brain" of the virtual power plant. The virtual power plant management and control platform comprehensively draws on the advanced concepts and design experience of mature Internet platforms, and combines the actual needs of grid business to form a system architecture of Internet + power business, with functions such as multi-form resource access, dispatching demand triggering, online monitoring and management, etc. The virtual operation calculation of power plant characteristics is realized, and the business flow and information flow are effectively connected. At the same time, many scientific research institutes continue to explore virtual power plant operation models, carry out research on revenue incentives and transaction mechanisms, establish virtual power plant operators, and then use contract energy management to guide users to actively participate in grid interactions, creating open sharing, active and convenient, A new business format with a win-win situation for all parties.

\section{Analysis of the development trend and significance of intelligence in the future energy field}

\subsection{Development trend}

Currently, the world is experiencing the fourth industrial revolution, and information technology is changing rapidly with the rapid development of big data, artificial intelligence, blockchain and other technologies. These emerging science and technology create opportunities for the development of the energy industry, and their deep integration with the energy industry is an important way to obtain low-cost technologies. Low-cost technologies will change the world's energy landscape and thus reshape the energy industry of the future. The energy industry is one of the first to benefit from digitalization. Power plants, coal mines and oil and gas fields have long been applying information technology such as computers to improve automation and management, and now the concepts of digital power plants, digital mines, digital oil fields and digital refineries have been proposed, which have greatly improved labor productivity and reduced labor costs. The current and future period is the era from digitalization to intelligence. Smart power plants, mines, oil fields and refineries will further optimize production processes and streamline organizations and personnel "unmanned management" of plants is the basic model, which will greatly change the industry ecology.

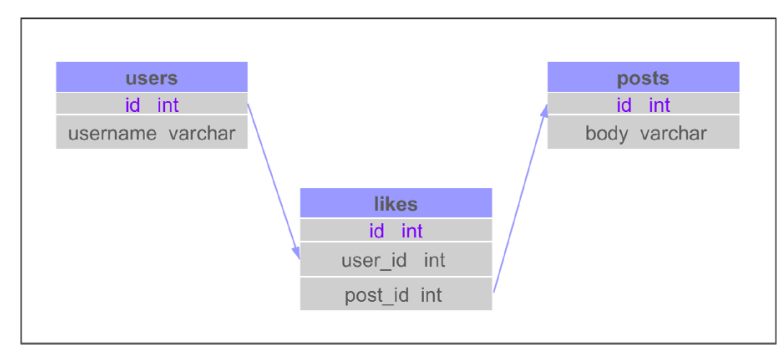

Figure 2. Intelligent database diagram 
The digitalization of energy is a major trend, and the innovative integration of digital technology and energy technology will enable the end-to-end visible, manageable and controllable intelligent management of energy infrastructure, from watts to watts + bits, from dumb equipment to intelligent systems, maximizing energy efficiency and shifting the world's energy development to green, clean and low-carbon. The world's energy development will also shift to green, clean and low-carbon. In the future, AI will be universally applied to the energy sector, replacing expert functions and enabling autonomous collaborative optimization of energy systems. In the future, digital energy will also move towards integration and simplification, including architectural integration, form simplification, and engineering productization, allowing equipment to change from more to less, from large to small, and gradually miniaturize, lighten, and modularize.

\subsection{Meaning analysis}

The rapid advancement of technologies such as artificial intelligence and blockchain will profoundly affect the development of future energy and, to some extent, will change the energy pattern and business conditions. Disruptive technology has always been accompanied by the history of human technological progress and has unforeseen properties. Only by accurately grasping these development trends can it be possible to make more accurate judgments about future energy technology development trends, such as: energy digitization, ubiquitous green electricity, full-link efficiency, AI blessing, fusion minimalism, energy network automated driving, Integrated smart energy, smart energy storage system, etc. [5].

The trend of the global energy transition is to promote the development of the energy structure to lowcarbon, clean, and electrified terminal energy, and digital technology will play an irreplaceable role in this process. With the high integration of digital technology and the energy industry, intelligence will become a high-level stage of energy digital development. Specifically, it will be manifested in the following aspects:

- Intelligence promotes the digital transformation of energy into a new stage. Intelligent development can provide the energy industry with technology, experience and valuable information based on massive big data analysis and artificial intelligence, which can greatly improve the efficiency of various fields of energy, reduce costs, and improve the competitiveness of the energy industry.

- Intelligence is the development direction of energy digital technology iteration. Intelligence is a major trend in the development of global science and technology, and the transformation of the energy industry will be deeply affected by it.

- Intelligence will provide a brand-new user experience for the development of energy digitalization, and create personalized business needs suitable for customers of different levels in accordance with the different needs of energy system customers.

\section{Conclusion}

The state of energy development is of great significance to national policy formulation and corporate strategic planning. The development of energy technology will profoundly affect the future energy pattern. Nowadays, the era of the energy industry has begun to take shape, and it is bound to open up a more exciting and beautiful future. We also call for digitization and intelligence to make waves in the big era, let technology and nature dance together, and inject more powerful impetus into social and economic development. Therefore, in the next step, it is necessary to spare no effort to deliver the latest network energy solutions to the majority of ecological partners and enterprise users. At the same time, it must also give full play to the platform incubation role, continue to implement the "platform + AI + ecology" ecological strategy, and in-depth collaboration with global ecological partners. Work together to build a community of destiny oriented to the intelligent era and further contribute to the digital transformation of the energy industry.

\section{Acknowledgments}

This work was supported by Special project of the scientific research plan of the Education Department of Shaanxi Province under Grant No. 19JK0169, the Key Research and Development Program Projects of Shaanxi Province under Grant No. 2019SF-257, PhD Startup Fund of Shaanxi University of Technology under Grant No.SLGQD2017-06.

\section{References}

1. Chen X. (2020) The advancement of energy technology revolution requires technology, finance and policy to work together. International Finance, 6: 6-11.

2. Dai H. (2020) Ubiquitous Power Internet of Things Promotes my country's Energy Transition and Industry Innovation.Electrical Times, 1: 20-21.

3. Wang X, Liu D, et al. (2019) Operation mechanism and key technologies of virtual power plants under the ubiquitous power Internet of Things. Power System Technology, 43: 3175 -3183.

4. Bai Y, Zhao Z, et al. (2019) Design and implementation of PHP service deployment strategy for domestic operating systems. Computer Applications and Software, 36: 17-21.

5. Jiang W. (2019) Current status and development trend of energy intelligent management. Chemical Management, 8: 35. 